The ASTROPHYSICAL JOURNAL SUPPLEMENT SERIES, 90:719-728, 1994 February

(c) 1994. The American Astronomical Society. All rights reserved. Printed in U.S.A.

\title{
PARTICLE ORBITS, TRAPPING, AND ACCELERATION IN A FILAMENTARY CURRENT SHEET MODEL
}

\author{
BERNHARD KLIEM \\ Astrophysical Institute Potsdam, An der Sternwarte 16, D-14482 Potsdam, Germany \\ E-mail: bkliem@aip.de \\ Received 1993 March 8; accepted 1993 June 10
}

\begin{abstract}
Test particle orbits in the two-dimensional Fadeev equilibrium with a perpendicular electric field added are analyzed to show that impulsive bursty reconnection, which has been proposed as a model for fragmentary energy release in solar flares, may account also for particle acceleration to (near) relativistic energies within a fraction of a second. The convective electric field connected with magnetic island dynamics can play an important role in the acceleration process.
\end{abstract}

Subject headings: acceleration of particles - MHD — plasmas — Sun: corona — Sun: flares

\section{INTRODUCTION}

One of the central problems in solar flare research is the identification of the mechanisms which transfer a large fraction of the released energy to accelerated particles, mainly to mildly relativistic electrons ( $W \sim 10-100 \mathrm{keV}$ ), and produce sometimes highly relativistic electrons and ions within a time interval of the order of a second. It is well known that collisionless plasmas have a tendency to give a (generally small) fraction of the free energy, which is transformed during active phases, to groups of accelerated particles and that there are many ways the plasma can accomplish this. In the case of solar flares the theory is challenged first of all by the high fraction of released energy which appears to go to the accelerated particles. The amount of energy in accelerated particles can at present be inferred best from the hard X-ray (HXR) emission of flares. Specific event analyses based on the nonthermal thicktarget HXR model, which appears to be more consistent with the data than competing models (Dennis \& Schwartz 1989), resulted in estimates of the fraction of released energy residing in accelerated particles of order $\gtrsim 20 \%$ (Duijveman, Hoyng, \& Machado 1982); even higher estimates had been derived from pre-SMM data. Such estimates are subject to large uncertainties (see the detailed discussion in Wu et al. 1989), but they point generally to an energetically important role of nonthermal mildly relativistic electrons during the impulsive flare phase.

Closely related is the "electron number problem." In the thick target model, the HXR luminosity implies that the precipitation rate of $\gtrsim 20 \mathrm{keV}$ electrons is typically $\sim 10^{36} \mathrm{~s}^{-1}$ (Hoyng, Brown, \& Van Beek 1976). The total number of electrons accelerated during the impulsive flare phase is then $\sim 10^{37}-10^{38}$. This is of the same order as, or possibly even exceeds, the number of electrons initially available in the primary energy release volume $\left(<10^{28} \mathrm{~cm}^{3}\right)$, which is supposed to be located in the corona at densities $\sim 10^{9}-10^{11} \mathrm{~cm}^{-3}$. This problem must be resolved by invoking an effective refilling mechanism for electrons in the source or by placing the primary acceleration volume in the transition region or chromosphere. It indicates in any case that the acceleration mecha- nism acts on the bulk of the electron distribution, not only on the tail.

Also the observed timescales of particle acceleration are challenging the theory. Temporal structure in the production of mildly relativistic electrons is generally blurred during propagation to the dense, HXR-emitting layers, but in some events HXR spikes with durations (FWHM) as short as $45 \mathrm{~ms}$ have been detected (Kiplinger et al. 1983, 1984). This indicates that the particles are accelerated in a (possibly very large) number of "elementary release events" and that the acceleration time as well as the decay time of the accelerating agent can be shorter as $20 \mathrm{~ms}$. The radio emission of the fast electrons is even more indicative of a high fragmentation and millisecond timescales of flare energy release. Groups of type III radio bursts are a characteristic signature of the impulsive flare phase; they show that $\leqslant 10^{3}$ separate beams of mildly relativistic electrons can be produced (Aschwanden et al. 1990). The rise time of these bursts is $\sim 100$ ms near onset (in the decimetric range). Also, decimetric spike bursts are frequently seen during the impulsive flare phase, in particular in compact flares; they too are thought to be produced by mildly relativistic electrons through a collective plasma emission processprobably a cyclotron maser instability. Their number in a flare (up to $\sim 10^{4}$ ) suggests high fragmentation of energy release, their bandwidth $(\Delta f / f<0.02)$ suggests small source dimensions $(<200 \mathrm{~km})$, and their duration $(\sim 20-100 \mathrm{~ms}$, depending on observing frequency) suggests correspondingly short acceleration times (Benz 1985; Güdel \& Benz 1990). The possibility that the extreme temporal and spectral structure of decimetric spikes is due to modulation of the nonlinear emission process has not been ruled out, but the excellent correlation of the spike burst occurrence rate with the HXR flux strongly suggests that the spikes do reflect the temporal structure of the particle source (Aschwanden \& Güdel 1992).

There are strong requirements also on theories of ion and relativistic particle acceleration. The observation of prompt gamma-ray lines has shown that energetic ions $(W \approx 10 \mathrm{MeV}$ nucleon $^{-1}$ ) can sometimes be produced in less than $2 \mathrm{~s}-\mathrm{syn}$ chronous with HXR-emitting electrons at the temporal resolution of 2 s (Forrest \& Chupp 1983; Kane et al. 1986). 
The delay of relativistic ions ( $W \gtrsim 100 \mathrm{MeV}$ ) could be constrained to $<5 \mathrm{~s}$ in one of these events. The presence of relativistic electrons can in some events be inferred from the extension of the continuous bremsstrahlung spectrum up to $\sim 100 \mathrm{MeV}$. Also, in a number of these events have very short rise and decay (!) times (of $<2 \mathrm{~s}$ and $\leqslant 4 \mathrm{~s}$, respectively) been observed in the gamma-range, and again these spikes were simultaneous to the HXR burst (Rieger \& Marschhäuser 1990). On the other hand, in most cases the relativistic particles appear with a delay. There are long-duration gamma-ray events. Relativistic particles are produced in relatively small number of large or complex flare events. Therefore, a distinction has frequently been made between "bulk" acceleration of the mildly relativistic electrons and relativistic particle acceleration in flares. It is not clear at present whether different acceleration mechanisms operate in these two domains.

The particle spectra must be inferred from the HXR and gamma-ray spectra or from interplanetary particle observations. Both possibilities involve ambiguities, however. The HXR spectra generally appear to be composed of two powerlaw sections with a break at typically $100 \mathrm{keV}$ (the break energy rises from $\sim 30 \mathrm{keV}$ to $\gtrsim 100 \mathrm{keV}$ in some events), but in many events a thermal (or a composed, multithermal) spectrum with $T \gtrsim 10^{8} \mathrm{~K}$ can fit the observations as well (this ambiguity is mainly due to insufficient spectral resolution of HXR instrumentation). A power-law photon spectrum, $d J /$ $d E \propto E^{-\gamma}$, is produced by a power-law spectrum of radiating electrons, $d N / d W \propto W^{-\delta}$, with $\delta=\gamma-\frac{1}{2}$ (e.g., Lin 1975). This may differ from the accelerated particle spectrum due to evolutionary effects on the particle distribution between the acceleration site and the HXR source in the thick target model; for example, Lin (1975) finds a power-law spectrum at the acceleration site with $\delta=\gamma+1$. Typically, $\gamma \sim 2-3$ below the spectral break and $\gamma \sim 3-7$ above the break. Thus the value of $100 \mathrm{keV}$ may be considered as a typical maximum energy of the mildly relativistic electrons. The upper, generally rapidly declining part of the HXR spectrum can in some events be traced to much higher energies. In these cases the spectra typically become harder again above $\sim 400 \mathrm{keV}$. This slowly decreasing electron bremsstrahlung continuum has been detected up to $\sim 100 \mathrm{MeV}$, in particular in the so-called electron-dominated events (Rieger \& Marschhäuser 1990).

Solar flare electrons directly detected in interplanetary space also show typically a power-law spectrum with a sharp break at $W \lesssim 100 \mathrm{keV}$, similar to the HXR-producing electrons. The spectral index may differ from that derived from $H X R$ spectra, however, indicating a heterogeneous particle source or/and partial reacceleration in upper coronal layers ( Pan et al. 1984).

The spectra of relativistic ions are fitted best by Bessel functions, which supports theories involving stochastic acceleration or stochastic transport (e.g., Forman, Ramaty, \& Zweibel 1986). Maximum energies may reach $W_{\max , i} \sim 2.5 \times 10^{10} \mathrm{eV}$.

The observations clearly show that the principal acceleration mechanism of the impulsive flare phase must be able to put a large fraction of the available energy to electrons, act on the bulk of the thermal electron distribution, operate at subsecond time scales, accelerate ions simultaneously. The maximum energy to be explained is $\sim 10^{5} \mathrm{eV}$ (possibly even $\sim 10^{8}$ $\mathrm{eV}$ ) for electrons and $\geq 10^{7} \mathrm{eV}$ (possibly $\sim 2 \times 10^{10} \mathrm{eV}$ ) for protons. It has been proposed that the particles are energized at shock waves, in turbulent wave fields, or by dc electric fields, where each of these possibilities includes several variants. At shocks particles are accelerated due to diffusion, drift along the shock, and charge separation. Alfvénic, magnetosonic, lower hybrid, and Langmuir wave turbulence all can produce highenergy tails. Substantial dc electric fields are supposed to result from localized excitation of anomalous resistivity and from induction during magnetic field line reconnection. In this paper it is demonstrated that multiple small-scale reconnection events, which are characteristic of the impulsive bursty mode of reconnection in a high magnetic Reynolds number plasma (Leboeuf, Tajima, \& Dawson 1982; Priest 1985; Kliem 1988; Biskamp \& Welter 1989) and have been proposed as a model of fragmentary energy release in flares (Tajima et al. 1987; Kliem 1990, 1991), can account for several aspects of impulsive phase acceleration. These are, in particular, acceleration of particles out of the main body of the thermal distributionup to the maximum observed energies, simultaneous electron and ion acceleration, very short timescales, and fragmentation. There is nearly no doubt that the reconnection of magnetic field lines is a central element of the flare energy release, it is synonymous with the induction of electric fields, and one is tempted to suppose that it directly produces also the bulk of the accelerated particles. The efficiency of the process has, however, been questioned due to the difficulty of injecting the particles at a sufficient rate into the tiny diffusion region of a (usually considered) large-scale X-type reconnection geometry and because of the tendency of the particles to leave the vicinity of the X-line quickly (e.g., Melrose 1990; Forman et al. 1986). Here it is shown that these arguments are far less critical for multiple small-scale reconnection.

On the other hand, it is questionable whether by-products of the energy release such as shock waves, which definitely also contribute to particle acceleration, are able to put a substantial fraction of the flare energy to the particles as indicated by observations. We note some further difficulties of the competing mechanisms without giving a thorough discussion. Diffusive shock wave acceleration and acceleration by turbulent MHD waves require a seed population of sufficiently energetic particles. They do not effectively accelerate particles out of the main body of the distribution. The shock drift acceleration and acceleration by lower hybrid waves, while acting also on thermal particles, do not lead to electron energies as high as $W_{e} \sim$ $10^{5} \mathrm{eV}$. Any mechanism that rests upon turbulent wave fields requires a very high energy density in the waves in order that a significant fraction of the released energy goes to accelerated particles. Generally two wave modes must be excited to realize simultaneous electron and ion acceleration. Even if shock waves can form in less than a second, it remains doubtful whether they can also stop accelerating particles in less than a second. One has to keep in mind, however, that the observed spectral forms ( power laws, Bessel function spectra) naturally result from stochastic processes involving diffusion in real or in velocity space (diffusive shock acceleration and turbulent acceleration ). But it is possible that the shocks or the turbulent wave fields, whose excitation in the energy release volume is quite expected, do not produce the bulk of the fast particles but merely modify the spectra before the particles can escape. The supposed fragmentation of the energy release can lead to a similar effect: fast particles may traverse several accelerating/ 
scattering volumes, that is, "diffuse," in a fragmentary source before they produce HXR and gamma-ray emission in the lower atmosphere; the observed spectra may be averages over many single spectra from the elementary energy release regions, particularly for integration times of the order of a second or larger.

In the following sections the chosen geometry of a filamentary current sheet containing multiple X-lines is introduced, then test particle orbits within that geometry are analyzed, and finally the resulting maximum particle energies, spectral forms, pitch-angle distributions, and scalings are discussed.

\section{MODEL ASSUMPTIONS}

Numerical simulations by Biskamp (1986), Biskamp \& Welter (1989, 1990), and others suggested that multiple smallscale reconnection naturally occurs in dynamical high magnetic Reynolds number plasmas like the flaring solar corona. Due to weak magnetic diffusion, large-scale motions will generally stretch out field lines, creating long, narrow layers with nonparallel field lines on either side. The shear flow may first stabilize these current sheets, but if they become sufficiently long and thin, the tearing instability breaks the sheet into a chain of current filaments (magnetic islands). This configuration is again unstable, and in a dynamical and disturbed environment the coalescence instability occurs. Part of the kinetic and magnetic energy in the system is thus transformed to different scales, and another part is dissipated during the multiple small-scale reconnection phases which are driven by the tearing and coalescence instabilities. Both instabilities are driven by the attraction between parallel currents, but while the tearing instability ("TI") occurs only in resistive MHD, the coalescence instability starts with the approach of current filaments as an ideal MHD instability, followed by a resistive phase, in which the merging of the current filaments proceeds (Pritchett $\&$ Wu 1979). These two phases of the coalescence instability, which are more clearly distinguishable for high magnetic Reynolds numbers, will be denoted by $\mathrm{CI}_{1}$ and $\mathrm{CI}_{2}$, respectively. The characteristic timescales are, accordingly,

$$
\begin{gathered}
\tau_{\mathrm{TI}} \sim\left(\tau_{r} \tau_{\mathrm{A}}\right)^{1 / 2}=S^{1 / 2} \tau_{\mathrm{A}} \\
\tau_{\mathrm{CI}_{\mathrm{l}}}=\xi^{-1} \frac{L_{\mathrm{CI}}}{V_{\mathrm{A}}}, \quad \xi=\frac{u}{V_{\mathrm{A}}} \sim 0.1-1,
\end{gathered}
$$

where $\tau_{r}=4 \pi \sigma(L / c)^{2}$ and $\tau_{\mathrm{A}}=L / V_{\mathrm{A}}$ are the resistive and Alfvénic timescales based on the current sheet half width $L$, respectively, $S$ is the Lundqvist (magnetic Reynolds) number, $V_{\mathrm{A}}=B\left(4 \pi N_{e} M\right)^{-1 / 2}$-Alfvén velocity, $N_{e}$-particle density, $M$-proton mass, and $\boldsymbol{u}$ is the flow velocity. Here $\tau_{\mathrm{CI}_{1}}$ is essentially the Alfvénic timescale based on the distance between approaching current filaments, $L_{\mathrm{CI}}$, equal to the wavelength of the fastest-growing tearing mode. The theory of the TI predicts $L_{\mathrm{CI}} \sim S^{1 / 4} L$ (in the case without shear flow), but simulations indicated that also much smaller $L_{\mathrm{Cl}}$ are possible. In any case, with typical values of $S\left(\gtrsim 10^{8}\right)$, we have $\tau_{\mathrm{Cl}_{1}} \ll \tau_{\mathrm{TI}}$. The typical timescale of the resistive coalescence phase, $\tau_{\mathrm{CI}_{2}}$, depends strongly on the plasma parameters and is not well known for high $S$; since the rapidly approaching current filaments enforce reconnection during the $\mathrm{Cl}$ in general more strongly than external flows do so during the TI, $\tau_{\mathrm{Cl}_{2}}$ lies between $\tau_{\mathrm{TI}}$ and $\tau_{\mathrm{Cl}_{1}}$. In fact, in the favorable case of strongly peaked current density and compressible plasma (i.e., low plasma-beta and nearly antiparallel external magnetic field configuration), $\tau_{\mathrm{CI}_{2}} \approx \tau_{\mathrm{CI}_{1}}$ has been observed in simulations ( Tajima, Brunel, \& Sakai 1982; Bhattacharjee, Brunel, \& Tajima 1983). The coalescence of a current filament pair is supposed to start at a certain location and then to propagate along the filament pair with approximately Alfvén velocity. At any given point the process has the duration $\tau_{\mathrm{CI}_{1}}+\tau_{\mathrm{Cl}_{2}}$, but the total duration of the coalescence as seen by a remote observer is of order $\tau_{\|}=$ $L_{\|} / V_{\mathrm{A}}$. Here $L_{\|}$, the extent of the coalescence process along the filaments, is the quantity which should correspond to observational source sizes, for example, the estimated size of decimetric spike bursts $\left(\sim 2 \times 10^{7} \mathrm{~cm}\right)$. Unlike the $\mathrm{TI}$, the $\mathrm{CI}$ leads to collapse of the current sheet (or at least of sections of the sheet; Kliem 1988). The coalescence instability thus releases more energy than the tearing instability (Leboeuf et al. 1982), and it has a higher rate of energy release, which makes it a prime candidate for flare energy release. The energy release then occurs primarily during discrete "coalescence events" of current filament pairs in an extended sheet, that is, in a fragmentary manner. The energy goes to kinetic energy of the fluid, plasma heating, and groups of accelerated particles.

There are three different mechanisms of particle acceleration due to reconnection in a current sheet, which rest on the parallel, convective, and perpendicular space charge electric field components, respectively. In connection with the CI, all these mechanisms can produce energetic particles in a fragmentary manner on short timescales. We will therefore briefly discuss the three field components. In a sheared configuration, which is generally to be expected in the solar corona, magnetic field line reconnection implies the occurrence of perpendicular and of parallel electric fields. A characteristic property of the process is that the nonconvective components of the electric field, which contain all parallel components, and the convective electric field component, $\boldsymbol{E}_{\text {conv }}=-\boldsymbol{u} \times \boldsymbol{B} / \mathrm{c}$, are of comparable magnitude in a small neighborhood of the $X$-lines, the diffusion region, where the reconnection actually proceeds. However, depending on parameters, either the parallel or the perpendicular field component may be dominant if the whole area of dynamic development is considered. In this connection it is useful to distinguish between the cases of existence of classical, collision-dominated resistivity and of the excitation of anomalous resistivity by a kinetic current-driven instability. The latter case requires current densities $j>j_{\text {cr }}$ where the critical current density is of order $j_{\mathrm{cr}} \sim N e v_{\mathrm{ti}}$ and $v_{\mathrm{ti}}=\left(T_{i} / M\right)^{1 / 2}$ is the ion thermal velocity (see Papadopoulos 1979). Using Ampère's law, this condition corresponds to magnetic field inhomogeneity scales $L_{B} \equiv B / \partial_{x} B<l_{\text {cr }} \sim 4 \beta^{-1} r_{\mathrm{ci}}\left(r_{\mathrm{ci}}\right.$-ion cyclotron radius, $\beta=16 \pi N_{e} T B^{-2}$-plasma beta) and can be satisfied only in tiny parts of the flare volume; possibly a large number of such areas exist, however. Since the current-driven kinetic instabilities have growth times much below the magnetohydrodynamic timescales and tend to quench themselves in the nonlinear phase, anomalous resistivity may also occur isolated in time.

For the low classical resistivity of the solar corona, the coalescence instability involves transitory phases of flux pileup in regions adjacent to the diffusion regions around the $\mathrm{X}$-lines 
during its first phase, particularly if the plasma is compressible. The convective electric field then approaches its maximum value, $E_{0}=V_{\mathrm{A}} B / c$ (e.g., Tajima et al. 1982), and substantially exceeds the fields in the vicinity of the X-lines. In comparison, the maximum parallel electric field that can stably coexist with the plasma in the diffusion region below the threshold for excitation of anomalous resistivity is the Dreicer field, $E_{\mathrm{D}}=(e)$ $\left.\lambda_{\mathrm{D}}\right)^{2} \ln \Lambda$ where $\lambda_{\mathrm{D}}=\left(T_{e} / 4 \pi N_{e} e^{2}\right)^{1 / 2}$ is the Debye length and $\Lambda=(4 \pi / 3) N_{e} \lambda_{\mathrm{D}}^{3}$ is the plasma parameter. Writing $E_{\mathrm{D}} / E_{0}$ in terms of dimensionless parameters,

$$
\frac{E_{\mathrm{D}}}{E_{0}}=\frac{1}{4} \frac{c}{v_{\mathrm{ti}}} \frac{\ln \Lambda}{\Lambda} \beta
$$

shows that the Dreicer field remains much smaller than $\boldsymbol{E}_{\text {conv }}$ in the solar corona where $c / v_{\mathrm{ti}} \approx 2 \times 10^{3}, \Lambda \sim 10^{7}-10^{9}$, and $\beta \sim 10^{-4}-10^{0}$. If $\tau_{\mathrm{Cl}_{2}} \gg \tau_{\mathrm{Cl}_{1}}$ (e.g., for very low resistivity and incompressible plasma), the rapid initial acceleration leads to an overshoot of magnetic pressure between the approaching filaments and subsequently to oscillations of the main flow pattern of the filament pairs during their slow merging, accompanied by reversals of $\boldsymbol{E}_{\text {conv }}$.

Alternatively, if the current density locally exceeds the kinetic instability threshold and anomalous resistivity is induced, substantial space charge electric fields can develop at that location because the underlying large-scale circuit has effectively been interrupted. This part of the electric field is directed along the current and has in general also parallel components. Possibly it forms double layers. Its strength is determined mainly by the parameters of the large-scale coronal circuit and is not bounded by $E_{0}$. If such strong parallel electric field components occur, they are expected to dominate the acceleration because the majority of particles cannot gain the full potential difference across the magnetic field due to deflection by the Lorentz force. Artificially induced dynamic reconnection has been observed to trigger the occurrence of anomalous resistivity near $X$-lines in the numerical experiments of Scholer \& Roth (1987); whether such an association also exists in the case of spontaneously occurring island coalescence will be the subject of a future investigation.

If the evolving magnetic field develops very small scales, $L_{B}<r_{\mathrm{ci}}$, charge separation of the magnetized electrons and the unmagnetized ions occurs, and the resulting electrostatic field across the magnetic field also contributes to particle acceleration (Tajima \& Sakai 1989). It is however seen from $l_{\mathrm{cr}} \sim 4 \beta^{-1} r_{\mathrm{ci}}$ that for low-beta plasmas like the solar corona the threshold of development of anomalous resistivity is reached much earlier. At that point triggering of the tearing instability is expected (Kliem 1988), which prevents the structure from further shrinking, and the resulting parallel electric fields will dominate the acceleration of particles.

In this paper particle acceleration by a perpendicular (essentially convective) electric field is selected because this component is always large during the coalescence instability, while the excitation of anomalous resistivity, albeit probable, is hypothetical. The convective electric field is generally considered as being unable to contribute to particle energization, but there are two important exceptions to the rule, relevant to the case under study:
1. At magnetic $X$ - and $O$-lines ( similar to the situation at magnetic inversion planes) particles perform a meander-like motion and decouple from the fluid.

2. Particles drift across the magnetic field due to inhomogeneities in $\boldsymbol{B}$; those particles that enter the region where an $\boldsymbol{E}_{\text {conv }}$ is induced in an inhomogeneous $\boldsymbol{B}$ from a region with a different local flow velocity $\boldsymbol{u}$ and are collisionless at the scales of the $\boldsymbol{u}$ and $\boldsymbol{B}$ variations experience energy gain or loss, respectively, depending on the sign of $\boldsymbol{E}_{\text {conv }}$.

It is our aim to demonstrate the principal possibility of rapid acceleration of a large number of particles to high energy. The qualitative properties of the mechanism and order of magnitude results can be obtained from a greatly simplified approach which considers test particle trajectories in an analytical model for the electromagnetic fields in a filamentary current sheet. We choose the well-known Fadeev equilibrium as magnetic field model. As a further simplification we adopt the configuration with vanishing magnetic guide field component because otherwise we cannot set up a model for the perpendicular electric field in a meaningful manner without detailed knowledge of the flow $\boldsymbol{u}$. (The Fadeev equilibrium and the antiparallel field case should also serve as a useful reference for later calculations based on a magnetohydrodynamical simulation of the CI.) The magnetic field is then given by

$$
\begin{aligned}
& B_{x}=-B_{0} \frac{\sinh (z / L)}{\cosh (z / L)+\epsilon \cos (x / L)} \\
& B_{z}=-B_{0} \frac{\epsilon \sin (x / L)}{\cosh (z / L)+\epsilon \cos (x / L)} .
\end{aligned}
$$

The parameter $\epsilon(0 \leq \epsilon<1)$ controls the current localization within the islands; its choice is $\epsilon=0.3$ throughout this paper which makes the island half width $z_{h} \approx L$. An electric field into the invariant direction is formally added to simulate the occurrence of convective electric fields due to filament motion during the coalescence instability. The simplest choice, $E_{y}=$ const implies a flow that is directed to, or away from, the center of the islands at each point, which is not characteristic of coalescence. Instead, neighboring islands approach each other as a whole; this is modeled by a periodic electric field $E_{y}(x)$ which changes sign at the position of the O-lines $(x / L \pi= \pm 1,3,5$, $\cdots)$. With $E_{y} \propto \cos (x / 2 L)$, acceleration and deceleration of particles trapped in the islands would cancel out. We take into account that the islands are deforming while approaching or repelling each other, with different magnitude of $|\boldsymbol{u}|$ and $|\boldsymbol{B}|$ on either side of the $\mathrm{O}$-lines. This results in a convective electric field that is asymmetric about the O-lines and may be modeled as (see Fig. 1)

$$
E_{y}=-E_{y 0} \cos \left(\frac{x}{2 L}\right) \cos ^{2}\left(\frac{x}{4 L}\right)
$$

This electric field permits net acceleration or deceleration of the trapped particles also. Our configuration (eqs. [3]-[5]) is not self-consistent ( since $\nabla \times E \neq 0$ implies $\partial B / \partial t \neq 0$ ), but it is expected to reveal the qualitative particle orbit characteristics (meander motion and drift motion ), on which the acceleration is based, correctly. Furthermore, the orbit calculations 

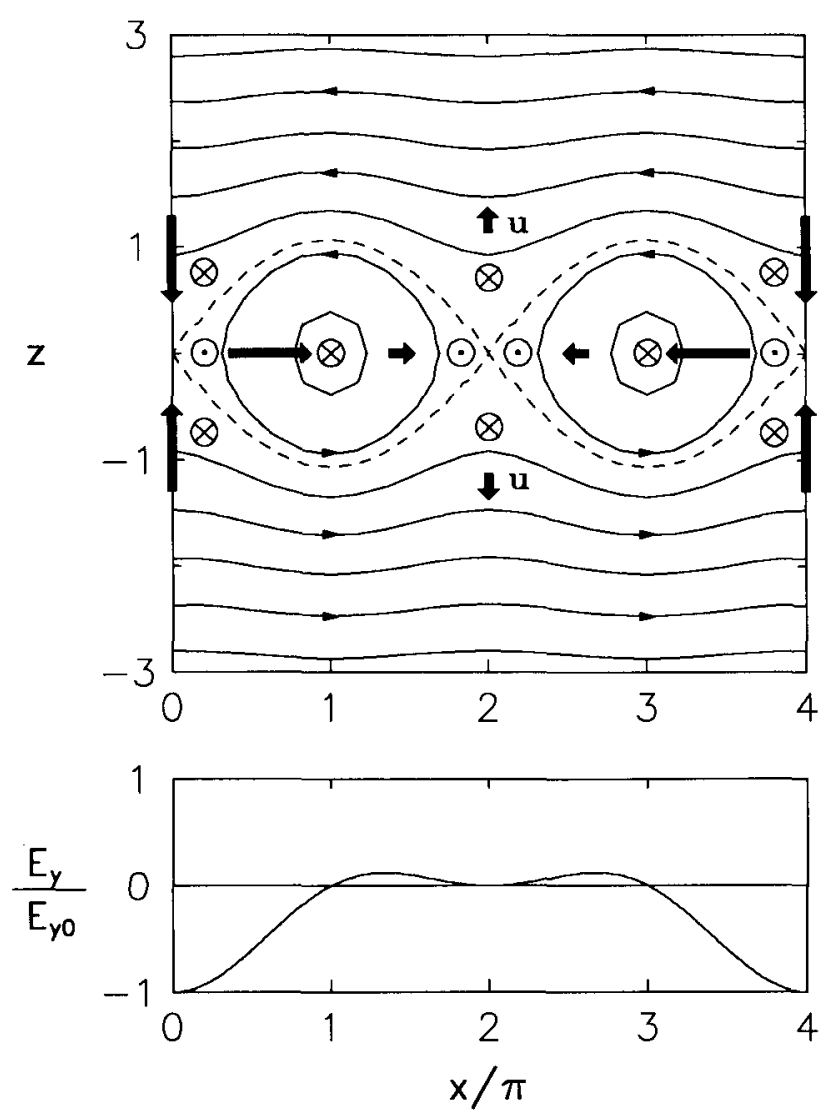

Fig. 1.-Considered configuration $\boldsymbol{B}(x, z), E_{y}(x)$ with the separatrices shown dashed. The implied flow $u$ ( equivalent to $V_{E}$ ) is indicated by heavy arrows. Arrows out of the plane give the direction of the $\nabla B$ drift for positive charges. Lengths are normalized to $L$ in this and subsequent figures.

show that the particles acquire a large portion of their final energy in a time interval much shorter than the timescale of magnetic field evolution, $\tau_{\mathrm{CI}_{1}}$.

A characteristic value of $L$ at which coronal current sheets typically undergo dynamic development, comprising island formation and coalescence, is not known. A lower limit is $L \geq$ $l_{\mathrm{cr}} \sim 4 \beta^{-1} r_{\mathrm{c}}$, where the localized excitation of anomalous resistivity triggers the tearing instability (Kliem 1988). Much smaller values follow from purely magnetohydrodynamic considerations (e.g., Biskamp 1986), but we do not consider these as realistic. The larger $L$, the longer $\tau_{\mathrm{TI}}$ and the more effective is the stabilization by the flow. Therefore we will use $L \approx 3 l_{\text {cr }}$ in numerical estimates, bearing in mind that slightly smaller but also larger values of $L$ are possible. For the plasma parameters in the region of primary flare energy release we adopt values of the lower solar corona, $N_{e}=10^{10} \mathrm{~cm}^{-3} ; T=2.5 \times 10^{6} \mathrm{~K}$ $(215 \mathrm{eV})$, and $B_{0}=200 \mathrm{G}$. This gives $L=2 \times 10^{4} \mathrm{~cm}$. Other parameters take the values $S=5 \times 10^{8}, \beta=4 \times 10^{-3}$, and $V_{\mathrm{A}}=4.4 \times 10^{8} \mathrm{~cm} \mathrm{~s}^{-1}$. The mean free path length becomes $l_{\mathrm{mfp}} \approx 4 \times 10^{6} \mathrm{~cm}$. This seems to invalidate a magnetohydrodynamic description at the considered spatial scale $L \sim l_{\text {cr }}$, but one should remember that the CI primarily involves fluid motions across the magnetic field where the freedom of the particles is restricted to $r_{\mathrm{ce}, \mathrm{i}} \ll l_{\mathrm{cr}}$. It is seen that even thermal parti- cles are collisionless over an island length ( even if $L_{\mathrm{CI}} \gtrsim S^{1 / 4} L$ ) and will have trajectories similar to the test particles considered below. Hence, bulk acceleration appears possible in the filamentary current sheet at spatial scales $L \gtrsim l_{\text {cr }}$. Kinetic energy of the fluid is transferred to particles via the convective electric field. The current carried by the accelerated particles, which is opposite to the original sheet current, $\boldsymbol{j}=(c / 4 \pi) \nabla \times$ $\boldsymbol{B}$, in the neighborhood of the X-lines (where the particles mainly perform gradient drift motions), will then modify the fluid dynamics and reduce the electric field strength. A selfconsistent treatment of these effects requires a particle simulation which is outside the scope of this paper.

Our parameter choices and $\xi=0.1-1$ lead to $E_{\text {conv }} \sim 10^{4}$ $10^{5} \mathrm{~V} \mathrm{~m}^{-1}, \tau_{\mathrm{Cl}_{1}}=0.3-3 \mathrm{~ms}$ ( for $L_{\mathrm{Cl}}=2 \pi L$ from eqs. [ 3,4$]$ ), and $\tau_{\|}=45 \mathrm{~ms}$ for $L_{\|}=2 \times 10^{7} \mathrm{~cm}$ (in accordance with spike burst observations).

Efficient acceleration of test particles by a perpendicular electric field was found earlier by Scholer \& Jamitzky ( 1987) for a similar configuration, a current sheet with two coalescing magnetic islands, which had been obtained from a magnetohydrodynamical simulation of the terrestrial magnetotail. In their model the strongest electric fields were connected with the development of anomalous resistivity at the "dynamic" $\mathrm{X}$-lines, yet still always directed perpendicular to the (twodimensional) magnetic field, and the strongest acceleration occurred at the dynamic X-lines. Here we wish to emphasize that also the convective electric field can efficiently accelerate particles in the filamentary current sheet, and we study the individual particle trajectories in more detail. In specifying plasma parameters we will concentrate on the solar flare case. For certain parameters our simplified model shows efficient particle acceleration also at the O-lines. Our approach differs from the calculations of Sakai (1992) primarily by the consideration of a multiple X-line geometry.

\section{TEST PARTICLE TRAJECTORIES}

We will first study the trajectories of single test particles. Taking $B_{0}, m V_{\mathrm{A}}, E_{0}=V_{\mathrm{A}} B_{0} / c, L, \tau_{\mathrm{A}}$ as units of magnetic field, momentum, electric field, distance, and time, respectively, the relativistic equations of motion of a test particle of charge $q$ and mass $m$ read in dimensionless variables

$$
\begin{aligned}
& \frac{d \boldsymbol{x}}{d t}=\frac{\boldsymbol{p}}{\Gamma} \\
& \frac{d \boldsymbol{p}}{d t}=\alpha(\boldsymbol{E}+\boldsymbol{p} \times \boldsymbol{B} / \Gamma),
\end{aligned}
$$

where

$$
\begin{aligned}
& \alpha=\frac{q B_{0} \tau_{\mathrm{A}}}{m c}=\frac{2 \pi}{T_{c}} \\
& \Gamma=\left(1+\left(V_{\mathrm{A}} / c\right)^{2} p^{2}\right)^{1 / 2} .
\end{aligned}
$$

The parameter $\alpha$ is equal to the normalized inverse cyclotron period of the considered particle species in the external field $B_{0}$, multiplied by $2 \pi$, and $\Gamma$ is the Lorentz factor. Our choice of plasma parameters and sheet width yields $\alpha=88$ for protons. 
These equations are integrated using an Adams-Moulton (predictor-corrector) scheme. Since the time step must be a fraction of the cyclotron period ( $\gtrsim 25$ time steps per cyclotron period are generally required), proton orbits were calculated in the majority of runs. The particle motion possesses chaotic properties, in particular for cyclotron radii $r_{c} \sim L$. Decreasing the time step further does therefore not avoid that some few particles, which passed (or were temporarily trapped) close to an X-line, reach completely different final positions and energies (just as slightly different initial conditions would cause). However, the basic parts of the particle trajectories on which the acceleration is based are independent of the partly chaotic behavior. Also, the properties of distributions of a large number of test particles, obtained in the following section, are reproducible if a different set of (randomly chosen) initial conditions is used and are not altered if the time step is further reduced.

In the given configuration there are three types of net motion in directions across the magnetic field: meander orbits at magnetic $X$ - and O-lines, the magnetic gradient and curvature drift, and the $\boldsymbol{E} \times \boldsymbol{B}$ drift.

Similar to the situation at uniform current sheets, those particles that reach the vicinity of a magnetic X- or O-line start performing a meander-like motion perpendicular to the magnetic field and decouple from the fluid motion. An electric field pointing into the direction of the meander accelerates or decelerates the particles. Independently of the sign of charge and the type of field reversal, collisionless particles are accelerated by the convective electric field if the underlying fluid velocity points toward the line or plane of magnetic field reversal (or rotation, if a guide field is present). In the opposite case the particles are decelerated. During meander motion the particle energy increases quadratically with time. The meander motion is stable at $\mathrm{O}$-lines if the electric field is accelerating $\left(E_{y 0} B_{0}>0\right.$ in our model): the particles continue the motion without limit, even if the magnetic field is slightly irregular or has different strength on both sides of the inversion. Formally this is due to the fact that while the particle energy is increasing during each semicircular part of the orbit on one side of the O-line, the Larmor radius is also increasing, and the particle must cross the O-line to repeat the process at the other side. In other words, the $\boldsymbol{E} \times \boldsymbol{B}$ drift (or the equivalent flow implicit in $\boldsymbol{E}_{\text {conv }}$ ) is fixing the particle's center of motion at the O-line. Of course, the implied flow can only temporarily occur at $\mathrm{O}$-lines in a compressible plasma, but the existence of stable meander orbits permits some particles to attain the full energy gain provided by the strength and extent of the convective electric field near the O-lines. (Note that this differs from the situation in magnetotail-like current sheets where a small normal magnetic field component $\left(B_{n}\right)$ causes the particles to leave the sheet after a finite interval of motion in the direction of the electric field, $\Delta y \sim 2 q B_{n} / m c$; e.g., Speiser 1965; Martens \& Young 1990.) On the other hand, only initially sufficiently energetic particles run into a meander-like motion about an O-line within one characteristic coalescence time and experience strong acceleration there, runs with a large number of test particles have shown that $r_{c} / L \gtrsim 0.2$ is required for rapid acceleration (less energetic particles do not "feel" much of the asymmetry of the electric field about the O-lines). Therefore, $\mathrm{O}$-line meander acceleration does not lead to bulk energization (as seems to be required for solar flares), but it may be of relevance to the most energetic particles observed in connection with dynamical plasma behavior.

At $\mathrm{X}$-lines, if a flow across the separatrices is present, the meander motion is not stable. (A steady electric field along the $\mathrm{X}$-lines is always connected with a flow across the separatrices, which is not necessarily true for electric fields induced by magnetic compression.) Generally after at most a few cyclotron periods with energy gain in the upstream region the test particles move to the downstream region where the electric field is decelerating during meander motion. There the particles are quickly lost from the vicinity of the $\mathrm{X}$-line and go through a phase of drift motion (where acceleration is again possible, see below). It is obvious from Figure 1 that acceleration during meander orbits at X-lines occurs in the configuration for either sign of $E_{y 0}$. Out of a large number of test particles only very few reach a meander orbit about an X-line (the majority crosses the separatrices earlier), but these acquire the highest final energy in the cases of negligible O-line acceleration.

Also the magnetic gradient and curvature drift of a particle with velocity $\boldsymbol{v}$, given by

$$
V_{\nabla}=\frac{m c}{2 q}\left(v_{\perp}^{2}+2 v_{\|}^{2}\right) \frac{\boldsymbol{B} \times \nabla \boldsymbol{B}}{B^{3}}
$$

is exactly parallel to $\pm E$ in the two-dimensional field case ( $B_{y}=$ $0)$. In the neighborhood of an $\mathrm{X}$ - or O-line, this drift motion is always oppositely directed to the meander motion about the $\mathrm{X}$ or O-line. Hence, in order to gain energy due to the drift along an $\mathrm{X}$ - or O-line, the orbit must be located in plasma that is streaming away from the $\mathrm{X}$ - or $\mathrm{O}$-line. Again it is obvious that this type of particle energization occurs near the $\mathrm{X}$-lines in the considered configuration (eqs. [3]- [5]) for either sign of $E_{y 0}$. (If a particle comes very close to an $\mathrm{X}$ - or $\mathrm{O}$-line, it might be expected that the particle first performs a nearly free motion (degenerate drift motion) for a finite interval along the line until its distance to this line is sufficiently large so that the drift motion follows, but for the majority of these particles a meander motion is initially observed instead.) Unless the particles are magnetically guided to return to the vicinity of an Xor O-line with an appropriately directed $\boldsymbol{E}_{\text {conv }}$, there will in general be only one period of strong acceleration due to the drift. In the filamentary current sheet, particles with orbits close to the separatrices are repeatedly guided into the neighborhood of the X-lines and experience several acceleration phases. Although the net energy gain decreases on average in each subsequent acceleration phase, the total energy gain due to the drift may exceed the energy gain during the phase of meander or nearly free motion. The effect of repeated phases of drift acceleration is somewhat overestimated in this paper due to the large asymmetry inherent in the electric field model; nevertheless, it is clear that multiple $\mathrm{X}$-line configurations permit higher final particle energies than single $X$-line configurations with similar electric field strengths.

The $E \times B$ drift, given by $\boldsymbol{V}_{E}=\left(\boldsymbol{E} \times \boldsymbol{B} / B^{2}\right) c$, is nothing else than the perpendicular component of the fluid motion in the particle picture, if no external electric field is applied. In our analytical model (eqs. [3]-[5]) it implies an artificial drift of the test particles across the (stationary) field lines. While this drift does not directly cause energy gain or loss, it influences 
the number of particles that start meander motion at an O-line and reach high final energy there. Our model thus overestimates $\mathrm{O}$-line acceleration if $E_{y 0} B_{0}>0$. This effect remains weak, however, because the $\boldsymbol{E} \times \boldsymbol{B}$ drift slows down in the neighborhood of the O-lines with our field model, equation (5), and the majority of the particles does not reach the vicinity of an O-line during the time of orbit integration which was always set equal to $\tau_{\mathrm{CI}_{1}}$.

Figure 2 illustrates the discussed characteristics of the single particle motion. Shown is a proton orbit which combines $\boldsymbol{E} \times$ $B$ drift toward the $\mathrm{X}$-line and finally toward the O-line, meander motion about the $\mathrm{X}$-line, and gradient drift motion along the $\mathrm{X}$-line. The particle is most rapidly accelerated ( similarly to a free motion) during the meander motion at the Xline (indicated by squares). Substantial acceleration also occurs during the drift at the first few passes close to the $\mathrm{X}$-line (indicated by triangles and stars). There are several drift acceleration phases of decreasing intensity due to magnetic guidance of the particle in the considered configuration. Shown is the first part of the orbit up to $t=0.4 \tau_{\mathrm{CI}_{1}}$. Subsequently the particle experiences strong $\mathrm{O}$-line meander acceleration (not shown here for clarity of the figure) to become the most energetic particle of the distribution shown in Figure 4, where its final position and energy at $t=\tau_{\mathrm{C}_{1}}$ can be seen. (The cross marks the position of the particle at the arbitrarily chosen time origin.)

The orbit characteristics reveal two advantages of the filamentary current sheet in comparison with single $\mathrm{X}$-line and magnetotail-like configurations in addition to the effect that there are many $X$-lines and that many particles have rapid access to an $X$-line. First, the maximum energy gain of the particles due to X-line acceleration is increased by the temporary trapping of the particles at field lines close to the separatrices and repeated guidance of the particles into the


FIG. 2.-Test particle orbit in the fields given by eqs. (3)-(5) which shows meander motion, $\nabla \boldsymbol{B}$ drift, and $\boldsymbol{E} \times \boldsymbol{B}$ drift. It is the first part of the orbit of the most energetic particle in the distribution shown in Fig. 4. See text for further explanation. Magnetic field lines in the $x-z$ plane are shown dashed. neighborhood of $\mathrm{X}$-lines so that several phases of gradientdrift-acceleration occur. Second, particles can be stably trapped and quasi-freely accelerated without limit during meander motion at the $\mathrm{O}$-lines.

\section{TEST PARTICLE DISTRIBUTIONS}

The relative importance of $\mathrm{X}$-line acceleration versus $\mathrm{O}$-line acceleration depends upon parameters like the initial particle energy and electric field strength. The final energy a particle reaches during $X$-line acceleration strongly depends upon the distance of closest approach of the particle to one of the $\mathrm{X}$ lines. To obtain an idea of the characteristic maximum energy of accelerated particle distributions for a range of parameters, runs were performed in which $\sim 10^{3}$ test particles were distributed randomly over the area of an island, $0 \leq x_{0} / L \leq 2 \pi,-1 \leq$ $z_{0} / L \leq 1, y_{0}=0$, and at the surface of a sphere in velocity space at $t=0$. The total time of orbit integration was always chosen to be $\tau_{\mathrm{CI}_{1}}$. The particle energy was varied between $7.65 \mathrm{eV}$ and $7.65 \mathrm{MeV}$ in steps of two orders of magnitude between the runs, for protons this corresponds to initial cyclotron radii of $\left(10^{-4}-10^{-1}\right) L$. The electric field strength was varied in the range $\pm E_{y 0} / E_{0}=\xi=10^{-1.5}-10^{0}$ in steps of one-half order of magnitude.

Results of such a calculation for a moderate field strength, $E_{y 0} / E_{0}=0.1$, and $E_{y 0} B_{0}>0$ are shown in Figures 3 and 4 . The mobility of the particles can be clearly seen (the difference between the two figures in this respect is mainly due to the different initial velocity and partly due to the larger mobility across the islands for larger cyclotron radii). Nearly all particles are trapped in the configuration. The first figure shows a dominating role of $\mathrm{O}$-line meander acceleration in the case of large cyclotron radius. For many different sets of parameter values, onset of O-line meander acceleration has been ob-
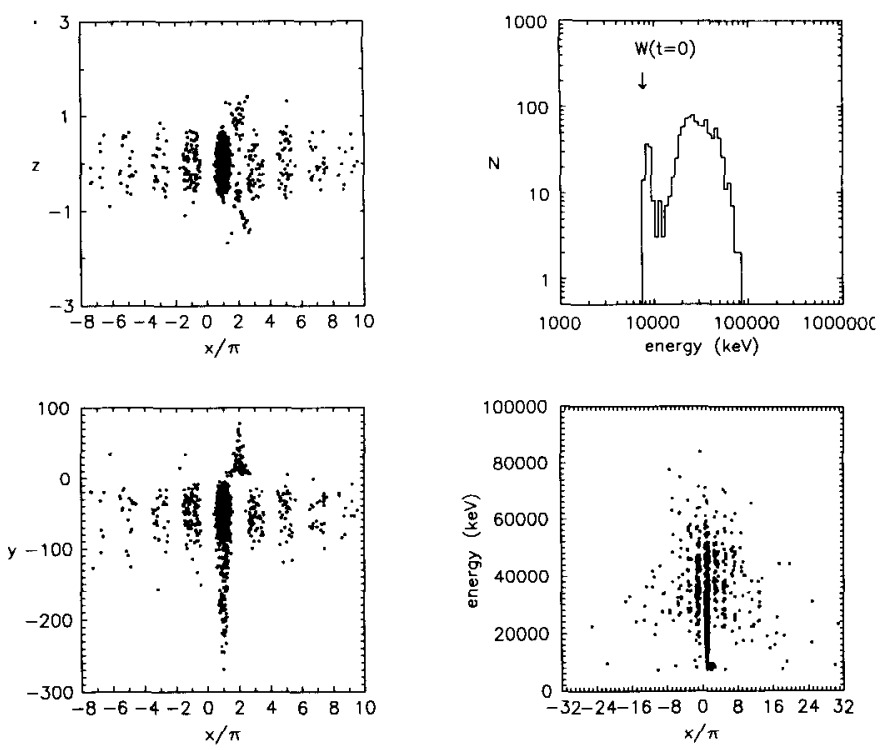

FIG. 3.-Final positions, energies, and the corresponding energy distribution of $10^{3}$ test particles (protons), which were initially monoenergetic and randomly distributed over the area of one island, after one coalescence time $\tau_{C c_{1}}$. Parameters are $E_{y 0} / E_{0}=0.1$ and $r_{c}(0) / L=0.1$ (i.e., $W(0) \approx$ $7.7 \mathrm{MeV}$ ). (Note that the more distant particles have been removed from the $z-x$ and $y-x$ scatter plots.) 

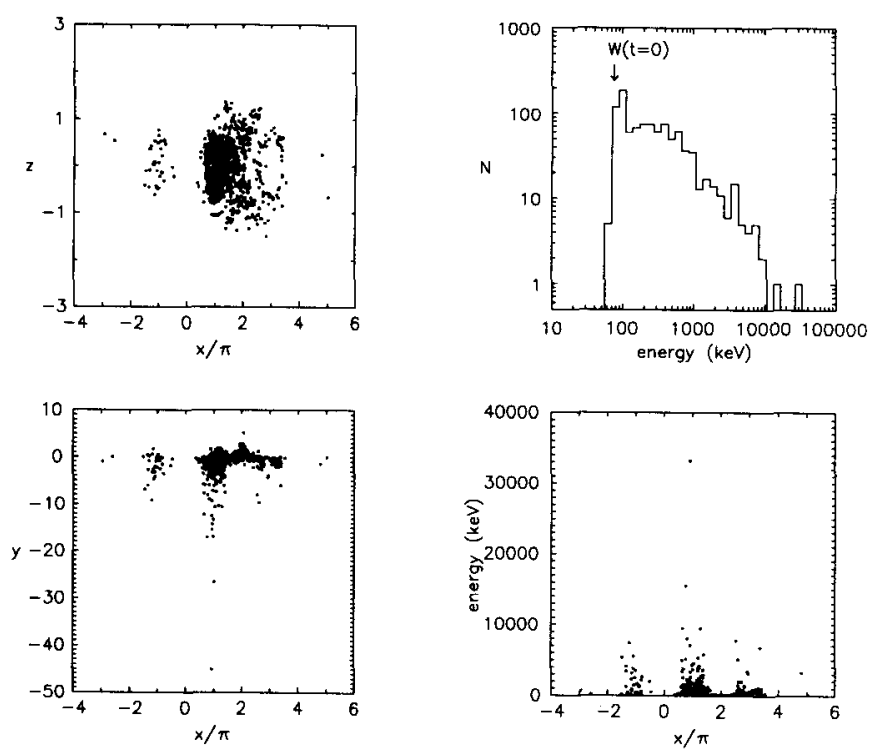
$\mathrm{keV})$.

FIG. 4.-Same as Fig. 3 but with $r_{c}(0) / L=0.01$ (i.e., $W(0) \approx 77$

served to occur whenever the individual cyclotron radius exceeds $r_{\mathrm{ci}} / L \gtrsim 0.2$, which corresponds to proton energies $W \gtrsim$ $2 \times 10^{7} \mathrm{eV}$ for the chosen sheet half width $L$. For the lower initial particle energy of Figure 4 and the chosen moderate electric field strength, only one particle acquires sufficient energy to experience appreciable O-line acceleration (the orbit of which is shown in Figure 2), hence this case is completely dominated by $\mathrm{X}$-line acceleration. The great majority of the $10^{3}$ particles do not come close enough to an X-line to perform meander motions there but are solely energized due to the drift along an X-line. In both cases a minority of particles can be seen to loose energy.

In the case of reversed electric field and plasma flow directions $\left(E_{y 0} B_{0}<0\right)$ a relatively similar picture is obtained for parameter values where in the former case $\mathrm{X}$-line acceleration is dominating ( there is no O-line meander acceleration in this case). This is apparent from the comparison of Figures 4 and 5. For otherwise identical parameters, the maximum final particle energy is clearly less in this case, since the effect of repeated X-line drift acceleration is less pronounced, but by not more than one order of magnitude. Also the number of accelerated particles is smaller. Many particles drift out of the sheet at even multiples of $2 \pi L$.

Characteristic maximum particle energies are shown in Figure 6 for $E_{y 0} B_{0}>0$ and a representative range of parameters. Since the actual maximum energy in a test particle distribution sensitively depends upon the details of the closest approach of one of the particles to an X-line, "characteristic" maximum energies ( obtained by disregarding the uppermost $0.5 \%$, respectively $1.0 \%$, of the final test particles) are given. It turns out that O-line meander acceleration is relevant if $r_{c}(t=0) / L \gtrsim$ 0.1 or $E_{y 0} / E_{0}=u / V_{\mathrm{A}} \approx 1$, otherwise X-line drift-acceleration is the relevant mechanism producing the most energetic particles. For our estimate of $L$ there are very few particles with $r_{c} / L \gtrsim 0.1$ in the corona. For dominating drift acceleration it is further found that the characteristic maximum particle energy
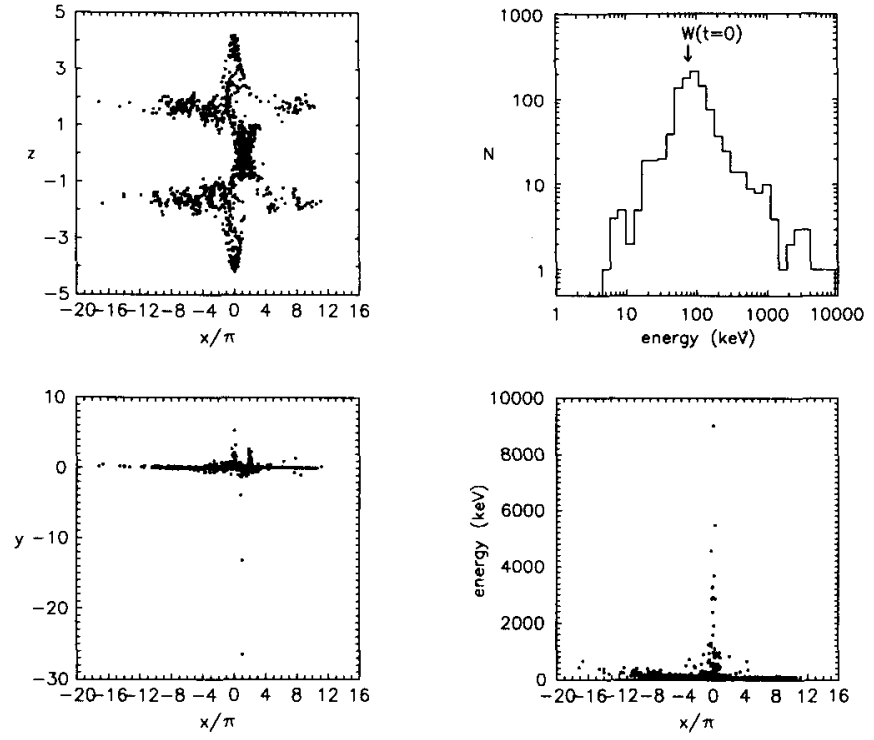

FIG. 5.-Same as Fig. 4 but with $E_{y 0} / E_{0}=-0.1$

scales roughly as

$$
W_{\max } \sim e \frac{L_{\mathrm{Cl}}}{2} E_{y 0}=\frac{u}{V_{\mathrm{A}}} e \frac{L_{\mathrm{CI}}}{2} \frac{V_{\mathrm{A}} B_{0}}{c}
$$

(with $L_{\mathrm{CI}}=2 \pi L$ in our model), as indicated in Figure 6 . A comparison with Figure 4 shows that, due to the repeated drift phases, many particles actually drift much more than an

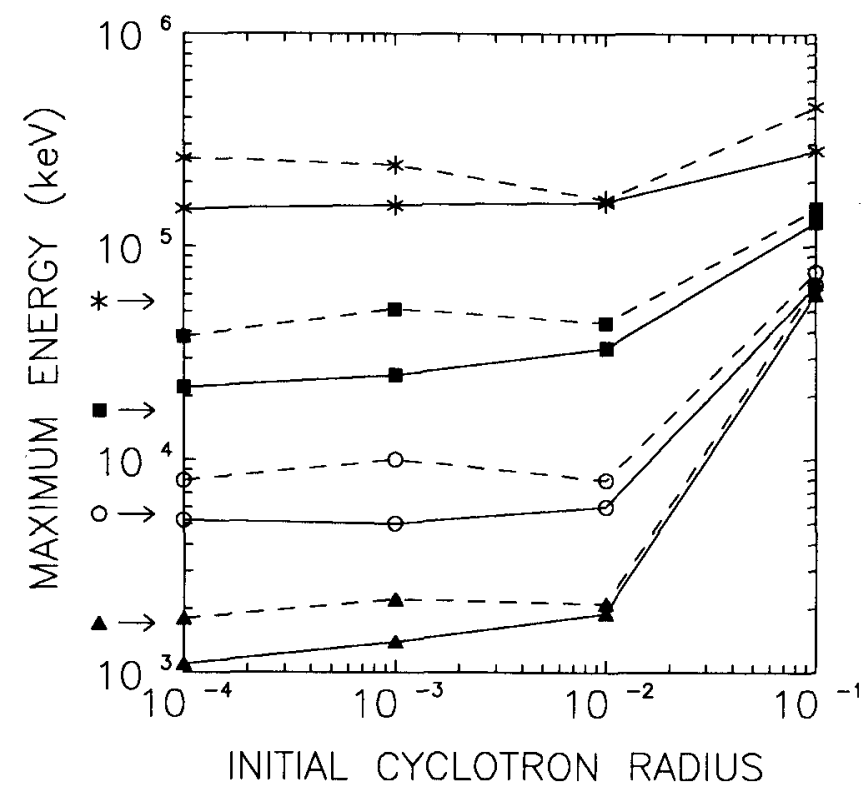

FIG. 6.-Comparison of "maximum" energies of runs with $10^{3}$ test protons each and different parameter choices with eq. (6), represented by arrows. Solid lines: energy reached by $1 \%$ of the test particles; dashed lines: energy reached by $0.5 \%$ of the test particles. Values of the applied electric field strength are $E_{y_{0}} / E_{0}=10^{0}$ (asterisks), $10^{-0.5}$ (squares), $10^{-1}$ (circles), and $10^{-1.5}$ (triangles). Cyclotron radii are normalized to $L$. 
island half length along the electric field, but the drift motion occurs in regions where $E_{y}(x)<E_{y 0}$. In the range of dominating drift acceleration it is seen from Figure 6 that there is only a weak dependence of the maximum energy on the initial energy; hence, with a distribution of initial particle energies, there would be no preferential acceleration of the superthermal over the thermal particles if both are collisionless.

Particles with smaller mass and cyclotron radius have a smaller probability to approach an X-or O-line close enough to start meander motion. Hence a decrease of the characteristic maximum energy with decreasing mass is expected, which is actually found but remains relatively weak (see Fig. 7).

The spectral forms of the accelerated test particle distributions are similar to the one shown in Figure 4 for those parameters where the X-line drift acceleration is dominating. The shape is only weakly dependent on the electric field strength in the relevant range $E_{y 0} / E_{0}=10^{-1.5}$ to $10^{-0.5}$, but the spectra extend over a broader energy range with smaller slope and a more and more pronounced high-energy drop for decreasing initial energy. The position of this high-energy drop does not depend much on the initial energy (see Fig. 6). These spectra result for initially monoenergetic particles. If the particles initially possess an energy distribution, a broken spectrum, slowly decreasing up to nearly the characteristic maximum energy that belongs to the given electric field strength and dropping then off rapidly, can be expected. Quantitative results such as spectral forms must be considered with caution due to the adopted model simplifications. Further, in a more general configuration, the simultaneous presence of many dynamical filaments in a large turbulent plasma volume as well as the excitation of waves by the rapidly moving filaments introduce a diffusive component into the particle motion and modify the escaping particle spectra accordingly.

Pitch angles of accelerated particles are given as a scatter plot in Figure 8 . Here the particles were initially again distributed

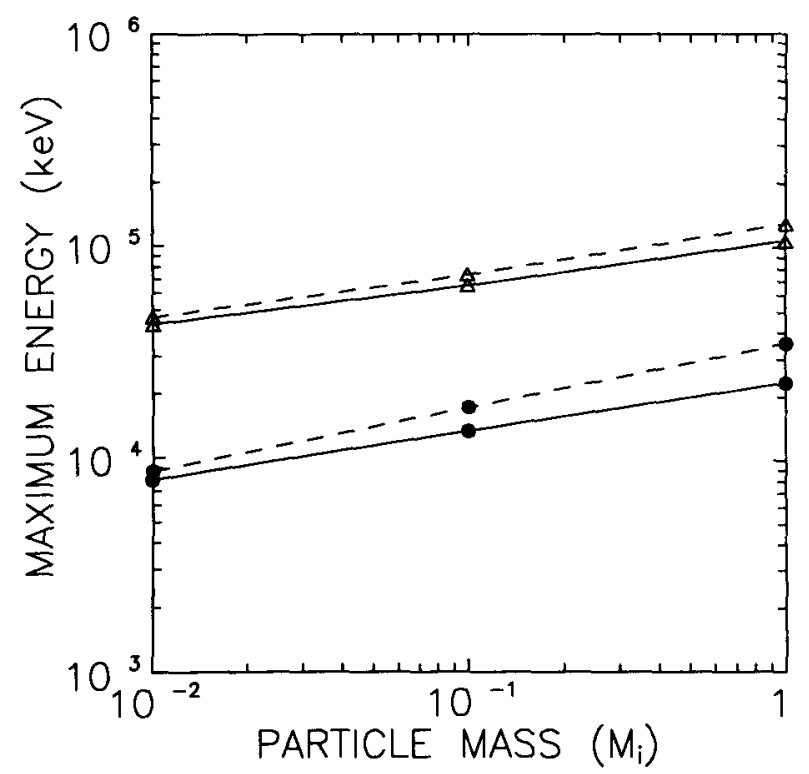

FiG. 7.-Dependence of the characteristic maximum particle energy on mass. Parameters are $E_{y 0} / E_{0}=10^{-0.5}$ and $r_{c}(0) / L=10^{-4}($ dots $), 10^{-1}$ (triangles). A good fit to the numerical values is given by $W_{\max } \sim(\mathrm{m} / M)^{\delta}$ with $\delta \approx \frac{1}{4}$.



Fig. 8.-Initial and final pitch angles and energies of $10^{3}$ protons after one coalescence time for $E_{y 0} / E_{0}=10^{-0.5}$ and $r_{c}(0) / L=10^{-3}$, marked by crosses and dots, respectively.

randomly but with uniform average density in the pitch angle interval $[0, \pi)$. Although the energization of the particles occurs in directions perpendicular to the magnetic field, the resulting pitch-angle distribution is nearly isotropic; only at relatively low and at the highest energies is a lack of small pitch angles discernible. This can be understood as follows. Close to the initial energy the cyclotron radius is much smaller than the radius of curvature, $r_{B}$, of the field lines and the perpendicular energization is reflected in the pitch angles. At higher energies $r_{c}>r_{B}$ holds, and the direction of the field lines varies considerably along a cyclotron orbit, leading to a nearly isotropic pitch angle distribution. The most energetic particles in Figure 8 (at $W \gtrsim 2 \times 10^{7} \mathrm{eV}$ ), having just entered a meander orbit, become confined more closely to an $\mathrm{O}$-line and to approximately perpendicular motion.

In our antiparallel field model the majority of the particles, which are trapped in the current sheet, move along the current filaments (i.e., in $y$-direction) only due to the $\nabla B$ drift. However, as soon as a guide field component is allowed, particle beams following the field lines (which spiral around the current filaments) are formed during a coalescence event since the particles also acquire substantial parallel velocity components within the acceleration region. The particles in these emanating beams possess comparable parallel and perpendicular velocity components. A similar picture is obtained for those highly energetic particles which are ejected from $\mathrm{O}$-line meander motion (even for $B_{y}=0$ ). This result differs from acceleration in magnetotail-like current sheets (Speiser 1965; Martens \& Young 1990) where the emanating particles possess small pitch angles.

\section{CONCLUSIONS AND DISCUSSION}

The small-scale dynamics of a current sheet connected with the tearing and coalescence instabilities and magnetic field line reconnection, which had earlier been proposed as a model for 
fragmentary energy release in flares, also leads to substantial particle acceleration. We have stressed the role of the perpendicular electric field which is always large during the coalescence of current filaments. Collisionless particles can be accelerated by the convective electric field induced in the filamentary current sheet.

Except for the case of very strong electric fields $(\xi \sim 1)$, $\mathrm{X}$-line drift acceleration is the dominating mechanism with maximum proton energies roughly scaling as $W_{\max } \sim e\left(L_{\mathrm{Cl}} /\right.$ 2) $E_{\perp}$ (in the considered case of antiparallel external fields), where $L_{\mathrm{Cl}}$ is the distance between the coalescing current filaments. The numerically found values of $W_{\max }$ decrease slightly with decreasing particle mass, leading to maximum electron energies that are smaller by nearly one order of magnitude. For typical parameters in solar flares this leads to $W_{\max , e} \sim 10^{6}$ $10^{7} \mathrm{eV}$ in accordance with gamma-ray observations during the impulsive phase. The corresponding acceleration times lie in the millisecond range. The accelerated particles occupy a broad range of pitch angles.

The presented model can account for several important properties of particle acceleration during the impulsive phase of solar flares, viz., bulk acceleration, fragmentation of the acceleration, timescales in the millisecond range, maximum energies in the $\mathrm{MeV}$ range. On the other hand, it is not yet able to explain preferential electron acceleration, the common appearance of power-law spectra, and the nearly uniform location of the spectral break in the range $\sim 30-100 \mathrm{keV}$. The main problem appears to be the ratio of electron to proton acceleration, which might be resolved in future by invoking some preheating of the electrons and larger spatial scales so that the electrons are already collisionless while the ions are not.

The obtained maximum energies may be somewhat optimistic since our simple analytical model, first, possesses a relatively strong asymmetry of the electric field strength about the $\mathrm{O}$-lines, giving rapid O-line meander acceleration and a strong influence of repeated drift acceleration near the X-lines, second, has the maximum electric field at the X-lines, not in their vicinity, which makes the initial phase of quasi-free acceleration at the X-lines more effective, and third, assumes antiparallel external magnetic fields. With a magnetic guide field component included, the particles can traverse the region of coalescence along the field lines that spiral around the current filaments. Thus the particles may stay in the acceleration region for an interval shorter than the coalescence time $\tau_{\mathrm{Cl}_{1}}$ used here. Moreover, the guide field, $B_{y}$, introduces a $z$-component of the $\nabla B$ drift. On the other hand, we have taken a very small value for $L_{\mathrm{CI}}$ in our estimates of $W_{\max }$. With the current sheet half width $L$ close to the limit $l_{\text {cr }} \sim 4 \beta^{-1} r_{\mathrm{ci}}$, the maximum particle energies are proportional to $B^{3}$ (if $\xi$ is independent of $B)$. Such a sensitive dependence seems to be unrealistic. Magnetohydrodynamical simulations of current sheets, which yield consistent electromagnetic fields, better estimates of $L$, and permit the inclusion of a guide field component, will lead to more reliable quantitative results for the proposed acceleration mechanism.

In comparison with a single $\mathrm{X}$-line configuration, a filamentary current sheet is far superior with respect to particle acceleration for the following reasons: (1) near-Alfvénic velocities and correspondingly strong electric fields occur near the Xlines; (2) many particles have rapid access to an X-line; (3) there are repeated drift acceleration phases at the X-lines due to trapping of the particles in the configuration; and (4) a small population of highly energetic particles can be produced by $\mathrm{O}$-line meander acceleration. The considered acceleration mechanism should be of general relevance to dynamical plasmas at high magnetic Reynolds numbers.

Discussions with M. Scholer and F. Jamitzky and comments by P. C. H. Martens are gratefully acknowledged. This work was supported by DARA under grant 50 QL 92085.

\section{REFERENCES}

Aschwanden, M. A., \& Güdel, M. 1992, ApJ, 401, 736

Aschwanden, M. A., Benz, A. O., Schwartz, R. A., Lin, R. P., Pelling, R. M., \& Stehling, W. 1990, Sol. Phys., 130, 39

Benz, A. O. 1985, Sol. Phys., 96, 357

Bhattacharjee, A., Brunel, F., \& Tajima, T. 1983, Phys. Fluids, 26, 3332

Biskamp, D. 1986, Phys. Fluids, 29, 1520

Biskamp, D., \& Welter, H. 1989, Phys. Fluids, B1, 1964 . 1990, Phys. Fluids, B2, 1787

Dennis, B. R., \& Schwartz, R. A. 1989, Sol. Phys., 121, 75

Duijveman, A., Hoyng, P., \& Machado, M. 1982, Sol. Phys., 81, 137

Forman, M. A., Ramaty, R., \& Zweibel, E. G. 1986, in Physics of the Sun, vol. 2, ed. P. A. Sturrock (Dordrecht: Reidel), 249

Forrest, D. J., \& Chupp, E. L. 1983, Nature, 305, 291

Güdel, M., \& Benz, A. O. 1990, A\&A, 231, 202

Hoyng, P., Brown, J. C., \& Van Beek, H. F. 1976, Sol. Phys., 48, 197

Kane, S. R., Chupp, E. L., Forrest, D. J., Share, G. H., \& Rieger, E. 1986, ApJ, 300, L95

Kiplinger, A. L., Dennis, B. R., Emslie, A. G., Frost, K. J., \& Orwig, L. E. 1983, ApJ, 265, L99

Kiplinger, A. L., Dennis, B. R., Frost, K. J., \& Orwig, L. E. 1984, ApJ, 287, L105

Kliem, B. 1988, in Reconnection in Space Plasma (ESA SP-285, Vol. 2), ed. T. D. Guyenne \& J. J. Hunt (Noordwijk: ESA), 117 1990, Astron. Nachr., 311, 399

1991, in Flares 22 Workshop, Dynamics of Solar Flares, ed. B. Schmieder \& E. R. Priest (Obs. de Paris), 119
Leboeuf, J. N., Tajima, T., \& Dawson, J. M. 1982, Phys. Fluids, 25, 784 Lin, R. P. 1975, in IAU Symp. 68, Solar Gamma-, X-, and EUV Radiation, ed. S. R. Kane (Dordrecht: Reidel), 385

Martens, P. C. H., \& Young, A. 1990, ApJS, 73, 333

Melrose, D. B. 1990, Aust. J. Phys., 43, 703

Pan, L.-D., Lin, R. P., \& Kane, S. R. 1984, Sol. Phys., 91, 345

Papadopoulos, K. 1979, in Dynamics of the Magnetosphere, ed. S.-I. Akasofu (Dordrecht: Reidel), 289

Priest, E. R. 1985, Rep. Prog. Phys., 48, 955

Pritchett, P. L. \& Wu, C. C. 1979, Phys. Fluids, 22, 2140

Rieger, E., \& Marschhäuser, H. 1990, in MAX '91, Workshop 3: Max ' 91 /SMM Solar Flares: Observations and Theory, ed. R. M. Winglee \& A. L. Kiplinger (Boulder: Univ. Colorado), 68

Sakai, J.-I. 1992, Sol. Phys., 140, 99

Scholer, M., \& Jamitzky, F. 1987, J. Geophys. Res., 92, 12,181

Scholer, M., \& Roth, D. 1987, J. Geophys. Res., 92, 3223

Speiser, T. W. 1965, J. Geophys. Res., 70, 4219

Tajima, T., Brunel, F., \& Sakai, J. 1982, ApJ, 258, L45

Tajima, T., \& Sakai, J.-I. 1989, Soviet J. Plasma Phys., 15, 519

Tajima, T., Sakai, J., Nakajima, H., Kosugi, T., Brunel, F., \& Kundu, M. R. 1987, ApJ, 321, 1031

Wu, S. T., et al. 1989, in Energetic Phenomena on the Sun, ed. M. R. Kundu et al. (Dordrecht: Kluwer), 377 